\title{
Virulence and drug susceptibility of Mycobacterium celatum
}

\author{
Lanfranco Fattorini, ${ }^{1}$ Lucilla Baldassarri, ${ }^{2}$ Yong-Jun Li, ${ }^{1}$ \\ Maria Grazia Ammendolia, ${ }^{2}$ Yuming Fan, ${ }^{1}$ Simona Recchia, ${ }^{1}$ \\ Elisabetta Iona ${ }^{1}$ and Graziella Orefici ${ }^{1}$
}

Author for correspondence: Graziella Orefici. Tel: +3906 49902333. Fax: +390649387112. e-mail:marella@iss.it

Laboratory of Bacteriology and Medical Mycology ${ }^{1}$ and Laboratory of Ultrastructures², Istituto Superiore di Sanitá, Viale Regina Elena 299, 00161 Rome, Italy

\begin{abstract}
The virulence and drug susceptibility of a clinical isolate of Mycobacterium celatum which showed smooth transparent (ST) and smooth opaque (SO) colonies were studied. While ST cells multiplied intracellularly and maintained their coccobacillary form in a human macrophage model of infection, SO cells formed long filaments and completely destroyed the phagocytes. In BALB/c mice, the ST variant, but not the SO variant, grew efficiently in the spleen, liver and lung. The ST variant was usually more resistant in vitro than the SO variant to drugs, with MIC values for clarithromycin (CLA), azithromycin (AZI), ciprofloxacin, sparfloxacin, amikacin, clofazimine, ethambutol and isoniazid being higher than those of the SO variant. In beige mice infected with the more highly virulent variant ST, CLA and AZI were the most active drugs in terms of viable count reduction in organs and mutant selection. Together, these observations indicate that the ST variant of $M$. celatum is a virulent form that can be efficiently inhibited in vivo by CLA and AZI.
\end{abstract}

Keywords: Mycobacterium celatum, macrophage infection, mice infection model, drug susceptibility

\section{INTRODUCTION}

Mycobacterium celatum was first described in 1993 as a new mycobacterial species biochemically indistinguishable from the Mycobacterium avium-intracellulare complex (MAC), with a mycolic acid pattern closely related to that of Mycobacterium xenopi (Butler et al., 1993). The infections caused by this organism were reported to occur mostly in persons with suppressed cell-mediated immunity, such as AIDS patients (Piersimoni et al., 1994, 1997; Gholizadeh et al., 1998; Bonomo et al., 1998; Bull et al., 1995; Tortoli et al., 1995; Zurawski et al., 1997), but infections also occurred in apparently immunocompetent hosts (BuxGewehr et al., 1998). Drug susceptibility testing showed that this organism was resistant to rifampicin (RMP), isoniazid (INH) and pyrazinamide (Butler et al., 1993), so that regimens similar to those administered in MAC-

Abbreviations: AMI, amikacin; AZI, azithromycin; CIP, ciprofloxacin; CLA, clarithromycin; CLO, clofazimine; EMB, ethambutol; INH, isoniazid; i.p., intraperitoneally; MAC, Mycobacterium avium-intracellulare complex; RFB, rifabutin; RMP, rifampicin; SO, smooth opaque; SPA, sparfloxacin; ST, smooth transparent. infected patients were usually used for the treatment of M. celatum infections (Piersimoni et al., 1997; Gholizadeh et al., 1998; Bonomo et al., 1998; Bull et al., 1995; Tortoli et al., 1995; Zurawski et al., 1997).

Some authors (Butler et al., 1993) reported that $M$. celatum colonies were predominantly small, smooth and dome-shaped, and that flat transparent colonies were rarely observed. In other studies (Tortoli et al., 1995), colonies were found to be polymorphic and similar to those of MAC and M. xenopi. Colonies of strains isolated from AIDS patients were reported to be transparent in the first period of growth but creamy white and pigmented after 8-12 weeks incubation (Bull et al., 1995). The factors influencing the change in colony morphology in mycobacteria are not known, but studies on antimicrobial susceptibility and pathobiological significance of the smooth transparent (ST) and smooth opaque (SO) variants of MAC have been reported (Reddy et al., 1996); in contrast, no information on the virulence and drug susceptibility of $M$. celatum colonial variants has been published.

The purpose of this investigation was to present data on the growth in vitro, ex vivo and in vivo of two colonial 
variants of a M. celatum strain isolated from an AIDS patient; in addition, the in vitro and in vivo susceptibility of the two morphotypes to antimicrobial agents is shown.

\section{METHODS}

Micro-organism and isolation of colonial variants. A Mycobacterium celatum strain (Cel 3) isolated from an AIDS patient was kindly provided by Dr C. Piersimoni (Piersimoni et al., 1997) and used throughout this study. When the strain was grown on Middlebrook $7 \mathrm{H} 10$ agar (Difco) at $37^{\circ} \mathrm{C}$ under $5 \% \mathrm{CO}_{2}$, it showed two colonial variants with ST and SO morphology. ST and SO colonies were reisolated on $7 \mathrm{H} 10$ plates and incubated for 1 week, then suspended in Middlebrook $7 \mathrm{H} 9$ broth (Difco) and stored at $-40{ }^{\circ} \mathrm{C}$ until use. Colonies were observed using a stereomicroscope or an inverted microscope; bacterial cells were stained by the Kinyoun method (Master, 1992).

Infection studies in human macrophages. Leukocyte buffy coats obtained from healthy donors were separated over Ficoll-Hypaque (Histopaque 1077; Sigma), as previously described (Fattorini et al., 1995). Briefly, suspensions of isolated peripheral blood mononuclear cells prepared in complete RPMI 1640 medium (Life Technologies) containing $10 \%$ heat-inactivated $\left(56^{\circ} \mathrm{C}, 30 \mathrm{~min}\right)$ fetal calf serum, $25 \mathrm{mM}$ HEPES and $2 \mathrm{mM}$ L-glutamine were adjusted to $1 \times 10^{7}$ cells $\mathrm{ml}^{-1}$ and distributed in $0.5 \mathrm{ml}$ volumes in 24 -well tissue culture plates. After $2 \mathrm{~h}$ incubation at $37^{\circ} \mathrm{C}$ under a humidified $5 \% \mathrm{CO}_{2}$ atmosphere, non-adherent cells were aspirated and the monocytes $\left(2-4 \times 10^{5}\right.$ per well $)$ were washed extensively with warmed medium without antibiotics and counted by the method of Nakagawara \& Nathan (1983). After $4 \mathrm{~d}$ incubation, cells showed macrophage morphology and $>98 \%$ of them were able to ingest neutral red. Monolayers were exposed to either ST or SO bacteria at a ratio of 500 or 50 c.f.u. per macrophage, respectively, incubated for $2 \mathrm{~h}$, and washed extensively to remove extracellular bacteria. Cell viability was determined by the trypan blue exclusion method. At various times, infected macrophages were lysed to determine the number of c.f.u., as previously described (Fattorini et al., 1995).

Transmission electron microscopy. Samples for electron microscopy observations were fixed with $0 \cdot 1 \mathrm{M}$ sodium-cacodylate-buffered $4 \%$ paraformaldehyde $/ 1.25 \%$ glutaraldehyde $/ 10 \mathrm{mM} \mathrm{CaCl}_{2}$ for $24 \mathrm{~h}$ at $4{ }^{\circ} \mathrm{C}$. Post-fixation with $1 \%$ $\mathrm{OsO}_{4}$ overnight at $4{ }^{\circ} \mathrm{C}$ was followed by dehydration through a graded series of ethanol solutions, 1:1 (v/v) ethanol and acetone, and pure acetone, and embedding in Spurr resin (Taab). In some experiments, macrophages were pulsed with cationized ferritin (Sigma) in RPMI 1640 medium at a final concentration of $0 \cdot 2 \mathrm{mg} \mathrm{ml}{ }^{-1}$ for $3 \mathrm{~h}$ at $37^{\circ} \mathrm{C}$ before infection (Hart \& Young, 1975). Sections obtained with an ultramicrotome MT-2B (LKB Pharmacia) were stained with uranyl acetate and lead citrate and examined with a Philips 208S transmission electron microscope.

Infection studies in mice. Male BALB/c mice were obtained from Charles River. Mice were infected intraperitoneally (i.p.) with $0.5 \mathrm{ml}$ of a bacterial suspension containing $1 \times 10^{7} \mathrm{ST}$ or SO c.f.u. At different time points, mice were killed by cervical dislocation and $4 \mathrm{ml}$ sterile PBS was injected into the peritoneum. The fluid was withdrawn and added to an equal volume of Middlebrook $7 \mathrm{H} 9$ broth. Suspensions were serially 10-fold diluted and plated onto 7H10 agar medium. The c.f.u. were counted after incubation of the plates for 10-14 $\mathrm{d}$ at $37^{\circ} \mathrm{C}$ in humidified air with $5 \% \mathrm{CO}_{2}$. The organs, collected under aseptic conditions, were suspended in 7H9 medium, ground in homogenizers, briefly sonicated, and the number of c.f.u. was determined.

MIC determination by the agar dilution method. MICs were determined by the twofold agar dilution technique using Middlebrook 7H10 agar. Inocula were prepared by suspending SO or ST colonies in Middlebrook $7 \mathrm{H} 9$ broth. Antibiotic-containing plates with drug concentrations ranging from 64 to $0.06 \mu \mathrm{g} \mathrm{ml}^{-1}$ were inoculated with $3 \times 10^{2}$ or $3 \times 10^{3}$ c.f.u. and incubated at $37^{\circ} \mathrm{C}$ in plastic bags for $14 \mathrm{~d}$. The MIC was defined as the lowest drug concentration at which no visible growth of the organism was observed.

Antimicrobial susceptibility in beige mice. Male beige mice $\left(\mathrm{C} 57 \mathrm{BL} / 6 / \mathrm{bg}^{\mathrm{j}} / \mathrm{bg}^{\mathrm{j}}\right.$ ) were obtained from Jackson Laboratories. Mice were infected i.p. with $0.2 \mathrm{ml}$ of a bacterial suspension containing $1 \times 10^{7}$ ST c.f.u. (Gangadharam, 1995; Fattorini et al., 1998). One day after infection, five mice were killed, and the organs were aseptically removed, homogenized in $1.5 \mathrm{ml}$ Middlebrook $7 \mathrm{H} 9$ broth and sonicated for $10 \mathrm{~s}$. To enumerate c.f.u., appropriate dilutions of the homogenates were plated onto Middlebrook 7H10 agar and, after 2 weeks incubation at $37{ }^{\circ} \mathrm{C}$ under a humidified $5 \% \mathrm{CO}_{2}$ atmosphere, colonies were counted. The remaining mice were randomly allocated to an untreated control group and eight drug-treated groups. Starting from $1 \mathrm{~d}$ after infection, RMP, rifabutin (RFB), clarithromycin (CLA), azithromycin (AZI), ethambutol (EMB), ciprofloxacin (CIP) and INH were administered orally by gavage five times weekly at the following concentrations: RMP, $10 \mathrm{mg} \mathrm{kg}^{-1}$ (Ji et al., 1994); RFB, $10 \mathrm{mg} \mathrm{kg}^{-1}$ (Ji et al., 1994); CLA, $100 \mathrm{mg} \mathrm{kg}^{-1}$ (Ji et al., 1994); AZI, $100 \mathrm{mg} \mathrm{kg}^{-1}$ (Cynamon \& Klemens, 1992); EMB, $100 \mathrm{mg} \mathrm{kg}^{-1}$ (Klemens et al., 1993); CIP, $40 \mathrm{mg} \mathrm{kg}^{-1}$ (Inderlied et al., 1989); INH, $50 \mathrm{mg} \mathrm{kg}^{-1}$ (Fattorini et al., 1998). Amikacin (AMI) was injected subcutaneously five times weekly at $100 \mathrm{mg} \mathrm{kg}^{-1}$ (Fattorini et al., 1998). c.f.u. in treated and untreated mice were determined on days 28 and 56 post-infection. Control untreated mice were injected with saline. To reduce the carryover effects of the drugs in organs, treated mice were killed $72 \mathrm{~h}$ after administration of the last dose of the treatment ( $\mathrm{Ji}$ et al., 1994). Statistical evaluation of the differences in c.f.u. between treated and untreated mice was done by the Student's $t$ test. $P<0.05$ was considered significant.

Enumeration of resistant mutants. Drug-resistant mutants were enumerated in the spleen suspensions from both drugtreated and untreated control mice on day 56. Besides the determination of the total number of c.f.u., $0.25 \mathrm{ml}$ of the undiluted suspensions was plated onto Middlebrook $7 \mathrm{H} 10$ agar plates containing drug concentrations corresponding to eight times the MICs. The frequency of drug-resistant mutants in the bacterial population was defined as the ratio between the c.f.u. numbers of the resistant mutants and the total c.f.u. number.

\section{RESULTS}

\section{Colony and cellular morphology}

The morphology of SO and ST colonies and the microscopic appearance of SO and ST cells after $7 \mathrm{~d}$ incubation on Middlebrook 7H10 agar are shown in Fig. 1. The SO variant displayed smooth dome-shaped opaque colonies (Fig. 1a) which developed a pale yellow pigmentation after 3-4 weeks incubation; the ST variant formed smooth transparent colonies (Fig. 1b) with 

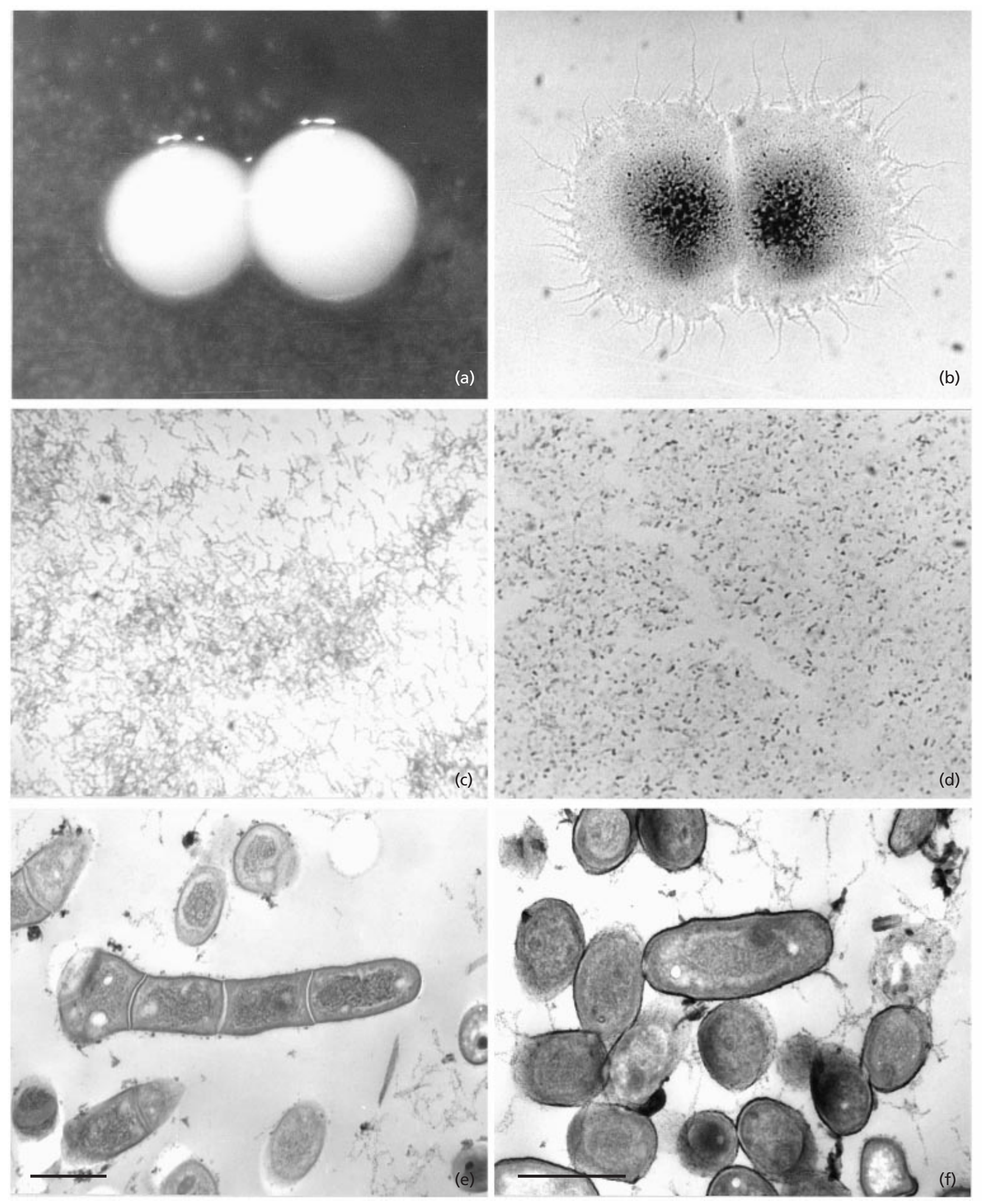

Fig. 1. Microscopic appearance of ST and SO variants of $M$. celatum. (a) SO colonies, as observed by a stereomicroscope. (b) ST colonies, as observed by an inverted microscope. (c) Microscopic aspect of SO cells (Kinyoun staining). (d) Microscopic aspect of ST cells (Kinyoun staining). (e) Electron micrograph of ultrathin sections of SO cells; bar, $0.5 \mu \mathrm{m}$. ( $\mathrm{f}$ ) Electron micrograph of ultrathin sections of ST cells; bar, $0.5 \mu \mathrm{m}$. 


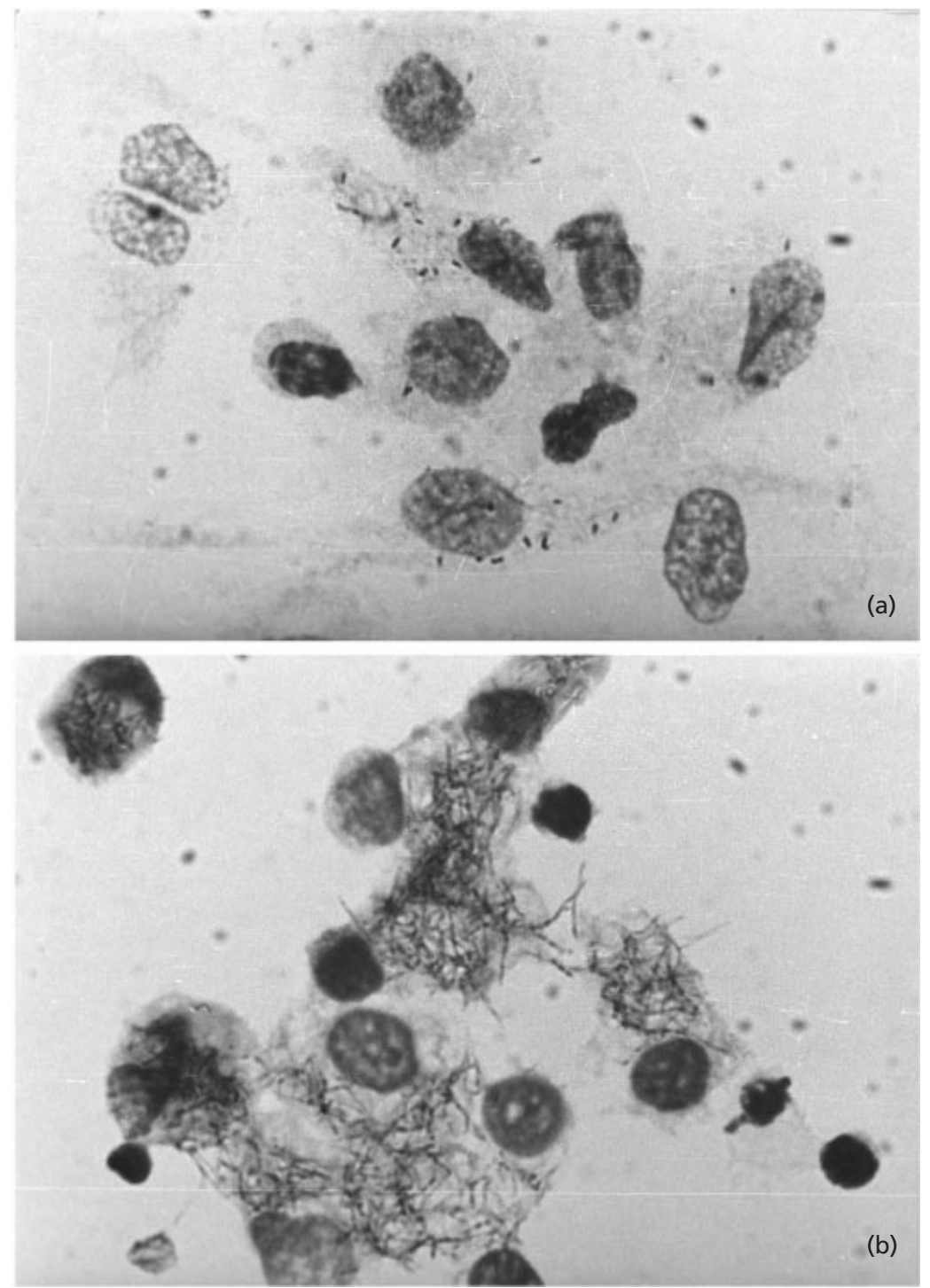

Fig. 2. Photomicrographs of human macrophages infected for $3 \mathrm{~d}$ with the ST (a) or SO (b) variant of $M$. celatum and stained by the Kinyoun method. irregular filamentous extensions at the edge. Microscopic examination of SO and ST colonies on day 7 showed that the SO variant was formed by aggregates of long branched septated cells (Fig. 1c) and the ST variant was formed by coccobacilli (Fig. 1d); after $14 \mathrm{~d}$ incubation, both ST and SO colonies were formed by cells similar to 7-d-old SO cells. While SO colonies were easily maintained in stable cultures in 7H10 medium, a transition of ST colonies into SO colonies $(\mathrm{ST}>\mathrm{SO})$ was observed after 3-4 weeks incubation, as shown by the appearance of opaque spots in the central area which increased in size and transformed ST into SO colonies with time.

By transmission electron microscopy, the majority of $\mathrm{SO}$ cells of 7-d-old cultures appeared as septated, sometimes branched, cells (Fig. 1e), while ST cells appeared as coccobacilli (Fig. 1f). In older cultures, ST cells also acquired a branched morphology similar to $\mathrm{SO}$ cells (results not shown).

\section{Growth of ST and SO variants in human macrophages}

The growth of the two colonial variants of M. celatum in human macrophages is shown in Fig. 2.

When macrophages were infected with ST cells, an increase from $1 \pm 0.3 \times 10^{5}$ c.f.u. (ml macrophage lysate $)^{-1}$ on day 0 (about one mycobacterium per macrophage) to $4 \pm 2 \times 10^{5}$ c.f.u. (ml macrophage lysate) $^{-1}$ on day 3 (three to four mycobacteria per macrophage; Fig. $2 \mathrm{a}$ ) and $13 \pm 3 \times 10^{5}$ c.f.u. (ml macrophage lysate) ${ }^{-1}$ on day 7 (about 10 mycobacteria per macrophage) was observed, as determined by both c.f.u. counts and Kinyoun staining. ST cells maintained their coccobacillary shape and did not alter the viability of the phagocytes (1,2 and $5 \%$ dead macrophages were seen on day 0,3 and 7 , respectively). The macrophages infected with the ST variant were about 30, 40 and $70 \%$ on day 0,3 and 7 , respectively, and the numbers of 
extracellular c.f.u. $(\mathrm{ml} \text { supernatant })^{-1}$ were $0 \cdot 1 \pm 0 \cdot 02 \times$ $10^{5}, 0 \cdot 4 \pm 0 \cdot 1 \times 10^{5}$ and $2 \pm 0 \cdot 4 \times 10^{5}$ on day 0,3 and 7 , respectively.

SO cells also multiplied efficiently within the macrophage, but the pattern of growth was different from that of ST cells. On day 0 , the number of intracellular c.f.u. was $2 \pm 0 \cdot 4 \times 10^{5}$ c.f.u. ( $\mathrm{ml}$ macrophage lysate $)^{-1}$, with about $50 \%$ of macrophages infected and one to three mycobacteria per macrophage; about $1 \%$ dead macrophages was seen and the c.f.u. number in the supernatant was $0.2 \pm 0.04 \times 10^{5} \mathrm{ml}^{-1}$. After $3 \mathrm{~d}$, a rapid growth of SO mycobacteria in macrophages in the form of long filaments invading the cytoplasm (Fig. 2b) and completely destroying the monolayers after $7 \mathrm{~d}$ was observed.

By electron microscopy it was shown that the bacteria were located exclusively inside phagosomes (Fig. 3). Usually these vacuoles contained some ST cells (from one to four) on day 0 (Fig. 3a) and a single bacterium on day 3 (Fig. 3b). Ferritin labelling (Fig. 3d) revealed that even though lysosome-phagosome fusion had occurred, ST cells showed no visible signs of degradation. The labelling also showed that the bacteria were surrounded by a 45-80-nm-thick electron-translucent zone resembling a capsule which separated the bacterial wall from the ferritin layer.

Single SO branched cells were observed inside separate vacuoles on day 0 or 3 (Fig. 3c). Ferritin labelling revealed that SO cells were surrounded by an irregularly shaped layer with short filaments protruding outward (Fig. 3e). No mycobacteria of either variant were apparently found outside vacuoles.

\section{Growth of ST and SO variants in BALB/c mice}

The kinetics of $M$. celatum growth in BALB/c mice is shown in Fig. 4. After i.p. infection, about $4 \%$ of the ST inoculum and $2 \%$ of the SO inoculum was recovered in the spleen, liver and lung on day 1 ; furthermore, from 6 to $7 \%$ of ST cells and $<1 \%$ of SO cells were found in the peritoneum. The ST variant multiplied in the spleen, liver and lung up to day 14, then a containment of the infection was observed. In infected mice, bacterial growth was associated with a $20 \%$ increase in the lung weight on day 7 , and $40 \%$ and $24 \%$ increase, respectively, in the spleen and liver weights on day 14 , in comparison with uninfected mice; after 2 weeks, organ enlargements slowly decreased but never returned to the uninfected mice levels. SO c.f.u. decreased by $80 \%$ in the liver and by $88 \%$ in the spleen and lung until day 14 , then slightly increased in the spleen and lung but not the liver; after this time, colonies with intermediate SO-ST morphology were observed in the viable count plates. SO variant infection was associated with a lower increase in organ weights up to day 14 , in comparison with ST variant infection (14\% increase in lung on day 7 and $29 \%$ and $10 \%$ increase in the spleen and liver weights, respectively, on day 14), followed by a rapid return to the uninfected mice levels. In the peritoneum, SO cells were cleared more rapidly than ST cells in the first day of infection, then a rapid decrease in ST and SO c.f.u. occurred.

\section{Antimicrobial susceptibility in vitro}

In vitro susceptibility of ST and SO variants to different drugs is shown in Table 1 . The ST variant was more resistant than the SO variant to CLA, AZI, CIP, SPA (sparfloxacin), AMI, CLO (clofazimine), EMB and INH, with MIC values ranging from $0 \cdot 25$ to $8 \mu \mathrm{g} \mathrm{m}^{-1}$ for the ST variant and from 0.06 to $0.5 \mu \mathrm{g} \mathrm{ml}^{-1}$ for the SO variant. The highest MIC differences between the ST and $\mathrm{SO}$ variants were observed for the fluoroquinolones CIP and SPA, with the ST variant being 32 and 16 times more resistant than the SO variant, respectively. Among other drugs, the ST variant was eight times more resistant than the SO variant to CLA, AZI and EMB, four times to CLO and INH, and two times to AMI. A noticeable exception was observed for the rifamycins RMP and RFB, for which the MIC values were 16 and $0.5 \mu \mathrm{g} \mathrm{ml}^{-1}$ for the ST variant, respectively, and 64 and $2 \mu \mathrm{g} \mathrm{ml}^{-1}$ for the SO variant, respectively.

\section{Antimicrobial susceptibility in vivo}

The antimicrobial susceptibility of ST variants to drugs in the beige mice model is shown in Fig. 5. The organisms efficiently multiplied in untreated control mice, as shown by a c.f.u. increase of approximately $3 \log _{10}$ in the spleen and liver and $2 \log _{10}$ in the lung. Compared with controls, all drugs tested caused a statistically significant c.f.u. reduction $(P<0.05)$ on day 28 and 56, with the exception of RMP and RFB in the lungs; however, as expected, RMP and RFB also showed poor activity in the spleens and livers (reduction of less that $2 \log _{10}$ in c.f.u. numbers on day 56). Although CIP was more effective that RMP and RFB, a slow and constant c.f.u. increase in all organs studied was observed on both day 28 and day 56. EMB was only bacteriostatic in the lung up to the end of the observation time but showed a better activity in the spleen and liver (more than $3 \log _{10}$ reduction in comparison with the control on day 56). AMI and INH showed an activity comparable with EMB in the spleen and liver, but with a remarkable regrowth in the lung after day 28 . AZI and CLA showed the best activity in all organs with $4 \log _{10}$ reduction on day 56 in the spleen and liver in comparison with controls, and more than $2 \log _{10}$ reduction in the lung. After $56 \mathrm{~d}$ infection, mutants with MIC values corresponding to eight times the wild-type ST MIC were observed in untreated and drug-treated mice, respectively, at the following frequencies: RMP, $1 \times$ $10^{-7 \cdot 18}$ and $1 \times 10^{-6 \cdot 6}$; RFB, $1 \times 10^{-7 \cdot 18}$ and $1 \times 10^{-6 \cdot 84}$; CIP, $1 \times 10^{-7 \cdot 39}$ and $1 \times 10^{-5 \cdot 68}$; EMB, $1 \times 10^{-6 \cdot 83}$ and $1 \times 10^{-4 \cdot 74} ; \mathrm{INH}, 1 \times 10^{-6 \cdot 55}$ and $1 \times 10^{-4 \cdot 29}$. No mutants were detected after therapy with CLA, AZI or AMI.

\section{DISCUSSION}

The existence of different colonial variants in mycobacteria was recognized a long time ago; however, the pathobiological significance of colony morphology has 

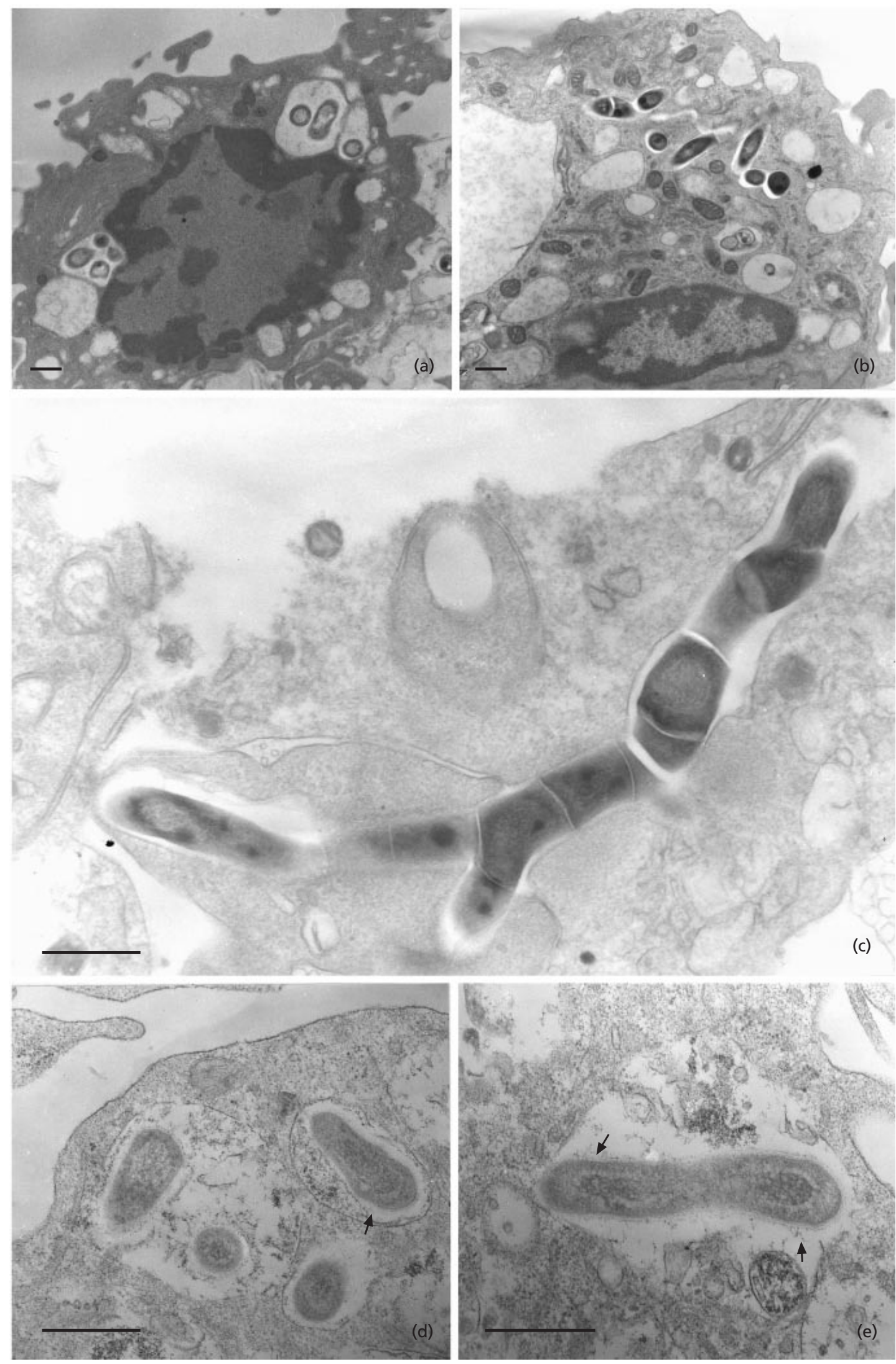

Fig. 3. Ultrathin sections of human macrophages infected with the ST ( $a, b, d)$ or SO (c, e) variant of M. celatum. The bacteria were confined inside phagosomes. Usually the vacuoles contained some ST bacteria (from one to four) on day 0 (a) and a single bacterium on day 3 (b). Ferritin labelling (d) showed the occurrence of phagosome-lysosome fusion but 

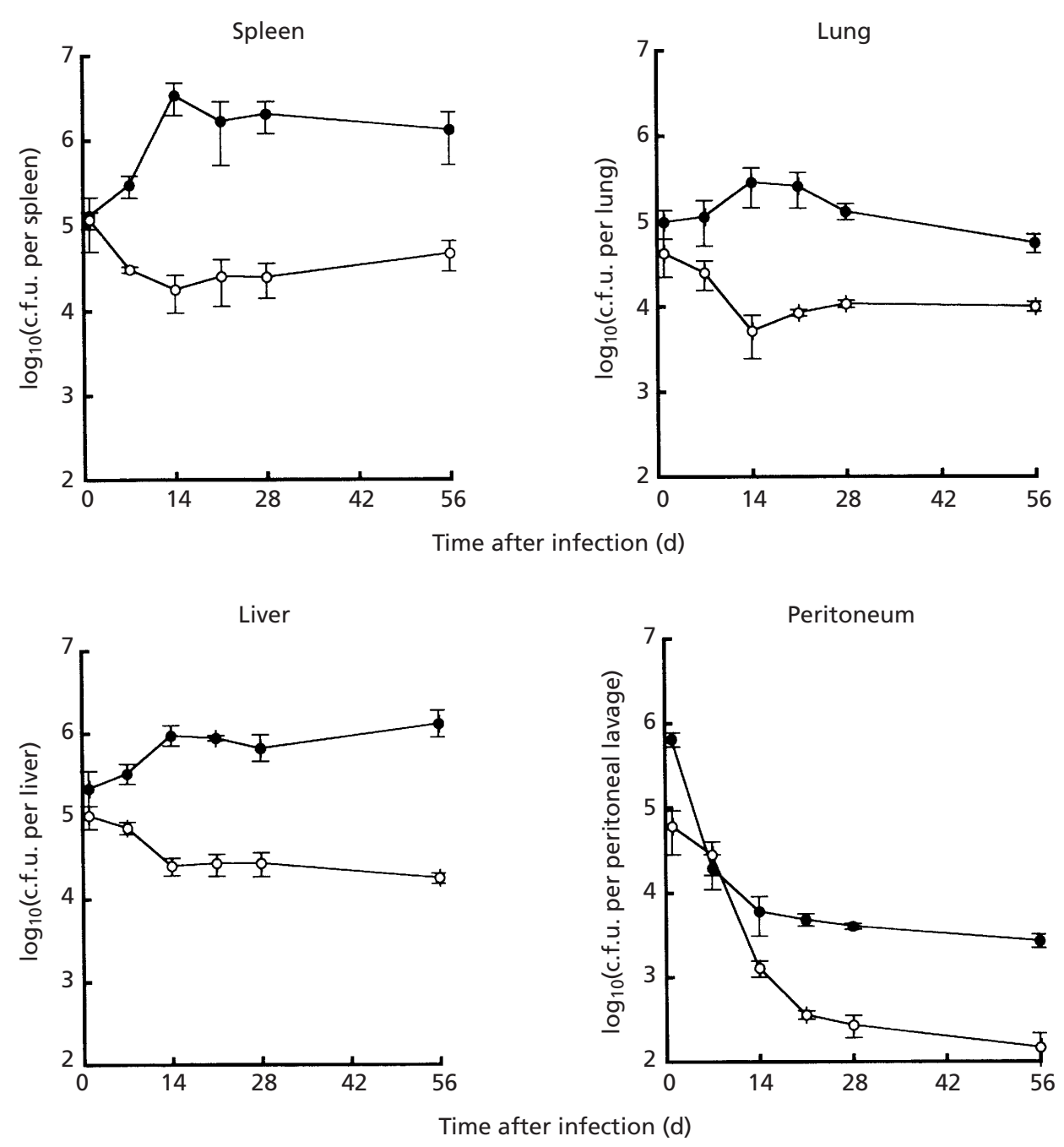

Fig. 4. Time course of ST and SO variants of $M$. celatum in the organs and peritoneal cavity of BALB/c mice inoculated i.p. with $10^{7}$ c.f.u. Each point represents the mean value of five mice and the standard deviation. 9 , ST variant; $\bigcirc$, SO variant.

not been extensively studied (Reddy et al., 1996; Schaefer et al., 1970; Rastogi et al., 1989).

The strain of $M$. celatum used in the present investigation showed SO colonies similar to those of MAC (Butler et al., 1993; Tortoli et al., 1995) and ST colonies with a 'moustached' appearance. In MAC, ST > SO transition has been attributed to various reasons, including metabolic starvation (McCarthy, 1974) and decreased temperature (Woodley \& David, 1976). The observation that in $M$. celatum ST $>\mathrm{SO}$ transition at $37^{\circ} \mathrm{C}$ occurred in the third to fourth week supports the possibility that also in this organism starving conditions favour the growth of SO cells, which are nutritionally less demanding than cells of the ST type.
When human macrophages were infected with ST cells, mycobacteria multiplied efficiently inside the phagocytes. A different pattern was displayed by SO cells, as shown by the formation of long filaments inside the macrophage which rapidly invaded and destroyed them. Similar observations were reported in MAC-infected HeLa cells (Brosbe et al., 1962), but, to our knowledge, not in MAC-infected macrophages, in which mycobacteria were found to lie inside the limits of the phagocytes (Meylan et al., 1990) and never exhibited a tendency toward branching (Crowle et al., 1986). The M. celatum pattern is more similar to that observed in cells infected with Nocardia asteroides, in which aggregates of filaments were formed in glial cell cultures 
Table 1. Activity of antimicrobial agents against ST and so colonial variants of $M$. celatum

\begin{tabular}{|c|c|c|}
\hline \multirow[t]{2}{*}{ Drug } & \multicolumn{2}{|c|}{$\operatorname{MIC}\left(\mu \mathrm{g} \mathrm{ml}^{-1}\right)$ of: } \\
\hline & ST variant & SO variant \\
\hline Clarithromycin & $1 \cdot 00$ & $0 \cdot 12$ \\
\hline Azithromycin & $4 \cdot 00$ & $0 \cdot 50$ \\
\hline Ciprofloxacin & $8 \cdot 00$ & $0 \cdot 25$ \\
\hline Sparfloxacin & $2 \cdot 00$ & $0 \cdot 12$ \\
\hline Amikacin & $1 \cdot 00$ & $0 \cdot 50$ \\
\hline Clofazimine & $0 \cdot 25$ & $0 \cdot 06$ \\
\hline Ethambutol & $4 \cdot 00$ & $0 \cdot 50$ \\
\hline Isoniazid & $2 \cdot 00$ & $0 \cdot 50$ \\
\hline Rifampicin & $16 \cdot 00$ & $64 \cdot 00$ \\
\hline Rifabutin & $0 \cdot 50$ & $2 \cdot 00$ \\
\hline
\end{tabular}

(Beaman \& Beaman, 1993) and human macrophages (unpublished observations of our group).

The ST variant was more virulent than the SO variant in the host, as reported for MAC (Reddy et al., 1996; Schaefer et al., 1970). In contrast to what was observed in cultured macrophages, the growth of SO bacteria was rapidly contained in mice, thus indicating the presence of an intact immune system is an essential requirement for SO variant control. However, the formation of intermediate ST-SO colonies which occurred during the progress of the infection in organs infected with the SO variant indicates that the ST phenotype is selected for the maintenance of infection. In comparison with what is known for MAC (Woodley
\& David, 1976), M. celatum shows an increased resistance to RMP and RFB. ST colonies are, in general, more resistant than SO colonies to many of the drugs tested; in contrast, it is interesting that in the $\mathrm{SO}$ variant the resistance to RFB and RMP was higher than in the ST variant.

Some discrepancies in the susceptibility of the clinical isolates of M. celatum have been reported (Piersimoni et al., 1997; Tortoli et al., 1995) in comparison with initial studies (Butler et al., 1993). This was believed to be due to either differences in the methods of testing or selection of strains representing different clones in the bacterial populations (Piersimoni et al., 1997; Tortoli et al., 1995). Our observations support the latter hypothesis and indicate that the presence of different proportions of $\mathrm{ST}$ and $\mathrm{SO}$ variants or intermediate forms in a bacterial population may affect drug susceptibility results; in vitro propagation or storage of clinical strains for an unspecified amount of time could be a cause of the differences in drug susceptibility results observed by various authors.

Therapy of M. celatum infections in AIDS patients is usually based upon administration of three to four anti-MAC agents, including CLA, AZI, RFB, CIP, AMI, EMB and CLO (Piersimoni et al., 1994, 1997; Gholizadeh et al., 1998; Bonomo et al., 1998; Bull et al., 1995; Tortoli et al., 1995; Zurawski et al., 1997; Masur, 1993). Our data showed that, in the beige mouse model, CLA, AZI and EMB are the most active antimicrobial agents among those tested, even if only a bacteriostatic activity was seen in the lung. However, while no drugresistant mutants could be detected in the spleen of mice treated with CLA and AZI, many mutants were found in
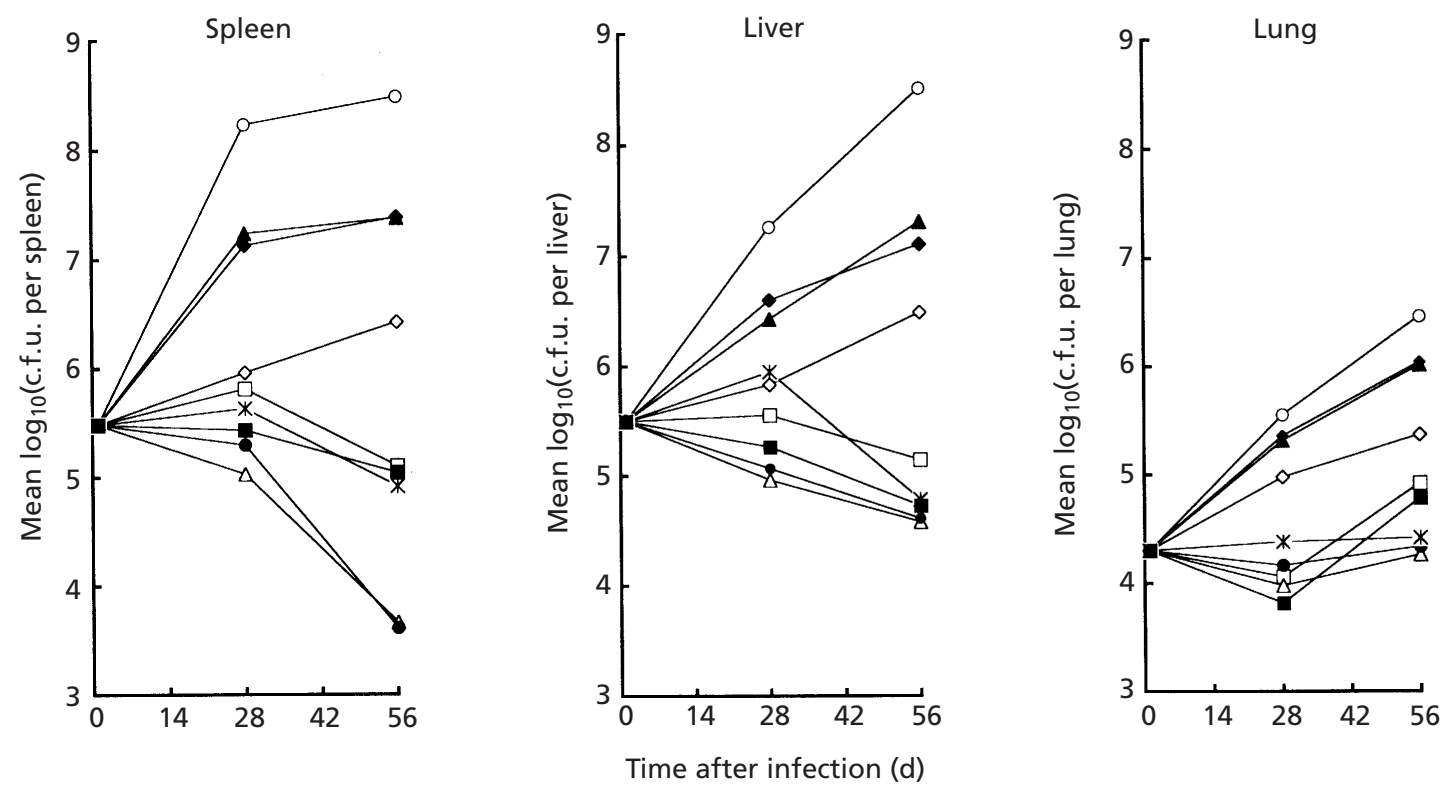

Fig. 5. Mean c.f.u. counts in organs of untreated and drug-treated beige mice infected with ST M. celatum. Standard deviations $(<20 \%)$ are not shown for clarity. Mice (five per group per time point) were inoculated i.p. with $10^{7}$ c.f.u. and treatments were begun 1 d later. $O, \mathrm{CTL} ; \square, \mathrm{INH} ; \triangle, \mathrm{CLA} ; 0, \mathrm{AZI} ; \times, \mathrm{EMB} ; \mathbf{\Delta}, \mathrm{RMP} ; \diamond, \mathrm{RFB} ; \diamond, \mathrm{CIP} ; \mathbf{\square}, \mathrm{AMI}$. 
EMB-treated mice. Overall, our data indicate that CLA and AZI, which are drugs recommended as first-choice agents for therapy of MAC infections in AIDS patients (Masur, 1993), could also be active against M. celatum infections in humans. The observation that CLAcontaining regimens were usually beneficial for treatment of these infections in HIV-positive patients (Piersimoni et al., 1997; Tortoli et al., 1995; Zurawski et al., 1997) is in keeping with our results. As expected, RMP, which showed high MIC values against both ST and SO variants, was not effective in vivo; unfortunately, RFB, which appeared to be more active than RMP in vitro, was also ineffective in mice. The latter observation is in keeping with a recent paper (Gholizadeh et al., 1998) in which the occurrence of $M$. celatum infection in two HIV-positive patients treated prophylactically with RFB was reported. As for CIP, this drug is considered to be active in vitro against $M$. celatum strains (Butler et al., 1993; Bonomo et al., 1998; Bull et al., 1995; Tortoli et al., 1995) but, unexpectedly, showed low efficacy in mice. The discrepancy may be explained with the knowledge that, in vitro, the ST variant is 32 times more resistant than the $\mathrm{SO}$ variant to CIP. These observations clearly emphasize the need to use freshly isolated colonies for testing in vitro drug susceptibility of $M$. celatum.

Overall, our results indicate that the two colonial variants, ST and SO, of M. celatum can show differential cellular morphology, growth in macrophages, virulence in mice and drug susceptibility, and that CLA and AZI are, in the beige mouse model, the most effective drugs against the virulent ST variant.

\section{ACKNOWLEDGEMENTS}

We thank Laura Parisi and Giovanna Alfarone, Istituto Superiore di Sanità, Rome, Italy, for valuable technical assistance. We also thank Dr Claudio Piersimoni, General Hospital "Umberto I", 60020 Torrette, Ancona, Italy, for providing the M. celatum strain used in this study. This work was supported in part by the Italian AIDS Project, Istituto Superiore di Sanità (grant 50/C/E).

\section{REFERENCES}

Beaman, L. \& Beaman, B. L. (1993). Interactions of Nocardia asteroides with murine glia cells in culture. Infect Immun 61, 343-347.

Bonomo, R. A., Briggs, J. M., Gross, W., Hassan, M., Graham, R. C., Butler, W. R. \& Salata, R. A. (1998). Mycobacterium celatum infection in a patient with AIDS. Clin Infect Dis 26, 243-245.

Brosbe, E. A., Sugihara, P. T. \& Smith, C. R. (1962). Growth characteristics of Mycobacterium avium and group III nonphotochromogenic mycobacteria in HeLa cells. J Bacteriol 84, 1282-1286.

Bull, T. J., Shanson, D. C., Archard, L. C., Yates, M. D., Hamid, M. E. \& Minnikin, D. E. (1995). A new group (type 3) of Mycobacterium celatum isolated from AIDS patients in the London area. Int J Syst Bacteriol 45, 861-862.

Butler, W. R., O'Connor, S. P., Yakrus, M. A. \& 8 other authors (1993). Mycobacterium celatum sp. nov. Int J Syst Bacteriol 43, 539-548.
Bux-Gewehr, I., Hagen, H. P., Rüsch-Gerdes, S. R. \& Feurle, G. E. (1998). Fatal pulmonary infection with Mycobacterium celatum in an apparently immunocompetent patient. J Clin Microbiol 36, 587-588.

Crowle, A. J., Tsang, A. Y., Vatter, A. E. \& May, M. H. (1986). Comparison of 15 laboratory and patient-derived strains of Mycobacterium avium for ability to infect and multiply in cultured human macrophages. Infect Immun 24, 812-821.

Cynamon, M. H. \& Klemens, S. P. (1992). Activity of azithromycin against Mycobacterium avium complex infection in beige mice. Antimicrob Agents Chemother 36, 1611-1613.

Fattorini, L., Li, B., Piersimoni, C., Tortoli, E., Xiao, Y., Santoro, C., Ricci, M. L. \& Orefici, G. (1995). In vitro and ex vivo activities of antimicrobial agents used in combination with clarithromycin, with or without amikacin, against Mycobacterium avium. Antimicrob Agents Chemother 39, 680-685.

Fattorini, L., Xiao, Y., Mattei, M., Li, Y., Iona, E., Ricci, M. L., Thoresen, O. F., Creti, R. \& Orefici, G. (1998). Activity of isoniazid alone and in combination with other drugs against Mycobacterium avium infection in beige mice. Antimicrob Agents Chemother 42, 712-714.

Gangadharam, P. R. J. (1995). Beige mouse model of Mycobacterium avium complex disease. Antimicrob Agents Chemother 39, 1647-1654.

Gholizadeh, Y., Varnerot, A., Maslo, C., Salauze, B., Badaoui, H., Vincent, V. \& Buré-Rossier, A. (1998). Mycobacterium celatum infection in two HIV-infected patients treated prophylactically with rifabutin. Eur J Clin Infect Dis 17, 278-281.

Hart, P. D. \& Young, M. R. (1975). Interference with normal phagosome-lysosome fusion in macrophages, using ingested yeast cells and suramin. Nature 256, 47-49.

Inderlied, C. B., Kolonowski, P. T., Wu, M. \& Young, L. S. (1989). Amikacin, ciprofloxacin, and imipenem treatment for disseminated Mycobacterium avium complex infection of beige mice. Antimicrob Agents Chemother 33, 176-180.

Ji, B., Lounis, N., Trouffot-Pernot, C. \& Grosset, J. (1994). Effectiveness of various antimicrobial agents against $\mathrm{Myco-}$ bacterium avium complex in the beige mouse model. Antimicrob Agents Chemother 38, 2521-2529.

Klemens, S. P., DeStefano, M. S. \& Cynamon, M. H. (1993). Therapy of multidrug-resistant tuberculosis: lessons from studies with mice. Antimicrob Agents Chemother 37, 2344-2347.

McCarthy, C. (1974). Effect of palmitic acid utilization on cell division in Mycobacterium avium. Infect Immun 9, 363-372.

Master, R. N. (1992). Mycobacteriology. In Clinical Microbiology Procedure Handbook, vol. 1, pp. 3.1-3.16. Edited by H. D. Isenberg. Washington, DC: American Society for Microbiology.

Masur, H. (1993). Recommendations on prophylaxis and therapy for disseminated Mycobacterium avium complex disease in patients infected with human immuno-deficiency virus. $N$ Engl J Med 329, 898-904.

Meylan, P. R., Richman, D. D. \& Kornbluth, R. S. (1990). Characterization and growth in human macrophages of Mycobacterium avium complex strains isolated from the blood of patients with acquired immunodeficiency syndrome. Infect Immun 58, 2564-2568.

Nakagawara, A. C. \& Nathan, C. F. (1983). A simple method for counting adherent cells: application to cultured human monocytes, macrophages and multinucleated giant cells. J Immunol Methods 56, 261-268. 
Piersimoni, C., Tortoli, E. \& De Sio, G. (1994). Disseminated infection due to Mycobacterium celatum in patient with AIDS. Lancet 344, 332.

Piersimoni, C., Tortoli, E., de Lalla, F., Nista, D., Donato, D., Bornigia, S. \& De Sio, G. (1997). Isolation of Mycobacterium celatum from patients infected with human immunodeficiency virus. Clin Infect Dis 24, 144-147.

Rastogi, N., Levy-Frebault, V., Blom-Potar, M. C. \& David, H. (1989). Ability of smooth and rough variants of Mycobacterium avium and $M$. intracellulare to multiply and survive intracellularly: role of C-mycosides. Zentralbl Bakteriol Mikrobiol Hyg A 270, 345-360.

Reddy, V. M., Luna-Heerera, J. \& Gangadharam, P. R. J. (1996). Pathobiological significance of colony morphology in Mycobacterium avium complex. Microb Pathog 21, 97-109.

Schaefer, W. B., Davis, C. L. \& Cohn, M. L. (1970). Pathogenicity of transparent, opaque, and rough variants of Mycobacterium avium in chickens and mice. Am Rev Respir Dis 102, 499-506.

Tortoli, E., Piersimoni, C., Bacosi, D. \& 11 other authors (1995). Isolation of the newly described species Mycobacterium celatum from AIDS patients. J Clin Microbiol 33, 137-140.

Woodley, C. L. \& David, H. L. (1976). Effect of temperature on the rate of the transparent to opaque colony type transition in Mycobacterium avium. Antimicrob Agents Chemother 9, 113-119.

Zurawski, C. A., Cage, G. D., Rimland, D. \& Blumberg, H. M. (1997). Pneumonia and bacteremia due to Mycobacterium celatum masquerading as Mycobacterium xenopi in patients with AIDS: an underdiagnosed problem? Clin Infect Dis 24, 140-143.

Received 10 April 2000; revised 10 July 2000; accepted 26 July 2000. 